\title{
Structure of two- and three-alpha systems in cold neutron matter
}

\author{
Hajime Moriya ${ }^{1, *}$, Hiroyuki Tajima ${ }^{2,3}$, Wataru Horiuchi ${ }^{1,}$, Kei Iida ${ }^{3}$, and Eiji Nakano ${ }^{3,}$ \\ ${ }^{1}$ Department of Physics, Hokkaido University, Sapporo 060-0810, Japan \\ ${ }^{2}$ Department of Physics, Graduate School of Science, The University of Tokyo, Tokyo 113-0033, Japan \\ ${ }^{3}$ Department of Mathematics and Physics, Kochi Univerisity, Kochi 780-8520, Japan
}

\begin{abstract}
We present stability and structure of two- and three-alpha systems embedded in dilute cold neutron matter. By solving a few-alpha Schrödinger equation with quasiparticle properties, i.e., effective mass and induced two- and three-alpha interactions, which are evaluated in terms of Fermi polarons, it is shown that ${ }^{8} \mathrm{Be}$ and the Hoyle state become bound at densities of about $10^{-4}$ and $10^{-3}$ of the saturation density, respectively. It is also seen that, under cold neutron matter environment, both systems become smaller than the corresponding systems in vacuum. Our results would affect astrophysical models for stellar collapse and neutron star mergers, as well as relevant reaction rates for nucleosynthesis.
\end{abstract}

\section{Introduction}

An alpha $\left({ }^{4} \mathrm{He}\right.$ nucleus) cluster plays an important role in the nuclear structure of light nuclei and astrophysical reactions relevant for the evolution of chemical elements in a burning star. In particular, the first $J^{\pi}=0^{+}$excited state of ${ }^{12} \mathrm{C}$, the so-called Hoyle state, is one of the most famous examples in which three alpha clusters are well developed. The Hoyle state works in such a way as to accelerate the triple-alpha reaction at low energies near the Gamow window [1], where the ${ }^{12} \mathrm{C}$ element is generated through sequential reactions via the resonant ground state of ${ }^{8} \mathrm{Be}$. It is important to describe such alpha-induced reactions accurately in the astrophysical explosive phenomena, e.g., core-collapse supernovae and neutron star mergers [2].

Recently, quasiparticle properties of an alpha particle in dilute cold neutron matter were discussed [3], where the alpha particle in the neutron matter was described as an impurity in a many-body background like a Fermi polaron in ultracold atomic physics [4]. In the polaron picture, the effective mass of the alpha particle is controlled by the interaction from the background. In this paper we discuss possible changes in the structure of two- and three-alpha systems in dilute neutron matter at zero temperature. By solving a few-alpha Schrödinger equation precisely, we discuss the stability and structure changes of the ground state of ${ }^{8} \mathrm{Be}$ and the Hoyle state in such neutron matter. In addition to the change of the effective mass, we introduce two- and three-alpha interactions induced by the neutron medium. Since all the details of the present evaluation are described in Ref. [5], in this paper, we briefly recapitulate those discussions and present some new results that were not presented there.

\footnotetext{
*e-mail: moriya@nucl.sci.hokudai.ac.jp
} 


\section{Method}

We employ a multi-alpha cluster model to describe the two- and three-alpha systems. The effective Hamiltonian for the three-alpha system is given by

$$
H=\sum_{i} \frac{p_{i}^{2}}{2 M^{*}}-T_{c m}+\sum_{i>j}\left(V_{\alpha \alpha, i j}+V_{\mathrm{eff}, i j}^{(2)}\right)+V_{\alpha \alpha \alpha}+V_{\mathrm{eff}}^{(3)},
$$

where $M^{*}$ is the effective mass of the alpha particle, $\boldsymbol{p}_{i}$ is the momentum operator of the $i$ th alpha particle, $T_{c m}$ is the kinetic energy of the center-of-mass motion, $V_{\alpha \alpha}$ and $V_{\alpha \alpha \alpha}$ are twoand three-alpha interactions, respectively. This two-alpha potential is deep enough to have some redundant or forbidden bound states by the Pauli principle between alpha particles. Details of the parameter set are given in Refs. [6, 7]. Physical states should be orthogonal to such forbidden states. These states are eliminated practically by using the projection method [8].

The effective mass of the alpha particle $M^{*}$ was given as a function of the Fermi momentum $k_{F}$ in Ref. [3]. Here, medium induced two- and three-alpha interactions ( $V_{\text {eff }}^{(2)}$ and $V_{\text {eff }}^{(3)}$ ) are also introduced by considering the simple Feynman diagrams in which few-alpha clusters exchange momenta. All the details are described in Ref. [5]. The wave function of the fewalpha system is described by a superposition of fully symmetrized correlated Gaussian basis functions whose variational parameters are optimized by the stochastic variational method $[9,10]$. All the numerical setups are detailed in Refs. [5, 11].

\section{Results and discussion}
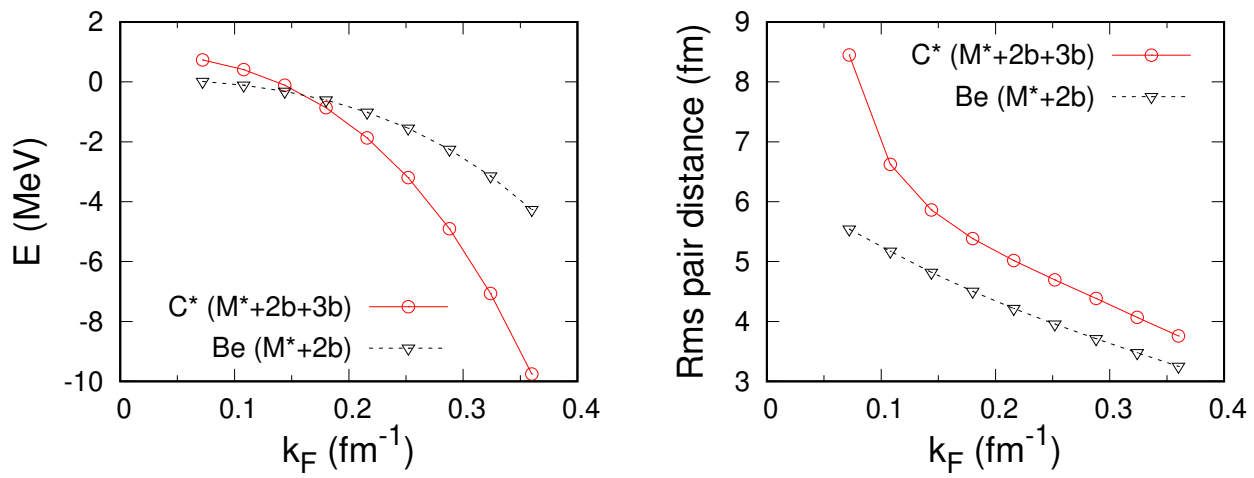

Figure 1. Energies (left) and root-mean-square pair distance (right) of the ground state of ${ }^{8} \mathrm{Be}$ and the Hoyle state $\mathrm{C}^{*}$ in neutron matter of the Fermi momentum $k_{F} . M^{*}, 2 b$, and $3 b$ denote calculations with the effective mass, induced two- and three-alpha interactions, respectively.

The alpha cluster environment is characterized by the Fermi momentum of background neutrons $k_{F}$, which affects the effective mass, induced two- and three-alpha interactions in the Hamiltonian. Here we discuss the energies and structure of the ground state of ${ }^{8} \mathrm{Be}$ and the Hoyle state as a function of $k_{F}$. The left panel of Fig. 1 plots the ground state of ${ }^{8} \mathrm{Be}$ and the Hoyle state energies as a function of $k_{F}$. The energies of both the ${ }^{8} \mathrm{Be}$ and the Hoyle state become lower for larger $k_{F}$ due to the short-range attraction of the induced two-alpha interaction. Eventually, the ground state of ${ }^{8} \mathrm{Be}$ becomes a bound state for $k_{F} \gtrsim 0.08 \mathrm{fm}^{-1}$ 
and the Hoyle state is also bound for $k_{F} \gtrsim 0.16 \mathrm{fm}^{-1}$, which correspond to approximately $10^{-4}$ and $10^{-3}$ of the saturation density $0.16 \mathrm{fm}^{-3}$, respectively. To see the structure of these alpha particle systems, we plot in the right panel of Fig. 1 the root-mean-square (rms) pair distance of the ground state of ${ }^{8} \mathrm{Be}$ and the Hoyle state. Both the ground state of ${ }^{8} \mathrm{Be}$ and the Hoyle state become smaller for larger $k_{F}$ monotonically. This shrinkage occurs because the internal amplitude of the wave function is strongly modified by the induced interactions mostly by the attractive induced two-alpha interaction. It is interesting to note that the size of the Hoyle state in the cold neutron matter shrinks as is consistent with the study of the finite system, e.g., the $\alpha-\alpha-n$ cluster calculation [12].

\section{Conclusion and prospects}

We have made precise calculations for few-alpha systems immersed in cold neutron matter. The alpha particle is treated as a polaron, i.e., quasiparticle dressed by excitations of background neutrons. We have found the possibility that the two- and three-alpha systems become bound in cold dilute neutron matter. The ground state of ${ }^{8} \mathrm{Be}$ and the Hoyle state become bound at $\approx 10^{-4}$ and $10^{-3}$ of the saturation density. The binding of these fundamental light nuclear ingredients would have a significant effect on the modeling of stellar collapse and neutron star mergers, as well as relevant reaction rates for nucleosynthesis. To realize these effects by the presence of the cold neutron matter in experiments, it is interesting to investigate $2 \alpha+X n$ and $3 \alpha+X n$ systems. For this purpose, it is desirable to relate alpha clusters interacting with a few neutrons and with infinite neutron matter. A detailed study along this direction is underway and will be reported elsewhere soon.

\section{Acknowledgement}

This work was in part supported by JSPS KAKENHI Nos. 17K05445, 18K03635, 18H01211, 18H04569, 18H05406, and 19H05140, and the Collaborative Research Program 2021, Information Initiative Center, Hokkaido University.

\section{References}

[1] F. Hoyle, Astrophys. J. Suppl. Ser. 1, 12 (1954)

[2] M. Oertel, M. Hempel, T. Klähn, and S. Typel, Rev. Mod. Phys. 89, 015007 (2017)

[3] E. Nakano, K. Iida, and W. Horiuchi, Phys. Rev. C 102, 055802 (2020)

[4] F. Chevy and C. Mora, Rep. Prog. Phys. 73, 112401 (2010)

[5] H. Moriya, H. Tajima, W. Horiuchi, K. Iida, and E. Nakano, Phys. Rev. C 104, 065801 (2021).

[6] C. Kurokawa, K. Katō, Phys. Rev. C 71, 021301 (2005)

[7] K. Fukatsu and K. Katō, Prog. Theor. Phys. 87, 151 (1992)

[8] V. I. Kukulin, and V. N. Pomenertsev, Ann. Phys. (N. Y.) 111, 333 (1978)

[9] K. Varga and Y. Suzuki, Phys. Rev. C 52, 2885 (1995)

[10] Y. Suzuki and K. Varga, Stochastic Variational Approach to Quantum-Mechanical FewBody Problems, Lecture Notes in Physics, Vol. m54 (Springer, Berlin, 1998)

[11] Lai Hnin Phyu, H. Moriya, W. Horiuchi, K. Iida, K. Noda, and M. T. Yamashita, Prog. Theor. Exp. Phys. 2020, 093D01 (2020)

[12] M. Lyu, Z. Ren, B. Zhou, Y. Funaki, H. Horiuchi, G. Ropke, P. Schuck, A. Tohsaki, C. $\mathrm{Xu}$, and T. Yamada, Phys. Rev. C 91, 014313 (2015) 